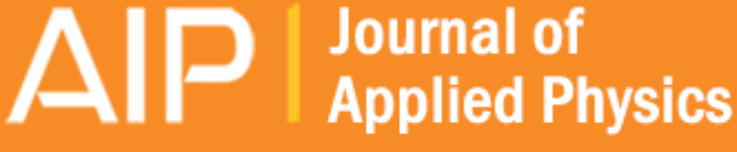

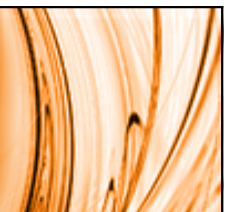

Fabrication and optical characteristics of phosphor-free InGaN nanopyramid white light emitting diodes by nanospherical-lens photolithography

Kui Wu, Tongbo Wei, Haiyang Zheng, Ding Lan, Xuecheng Wei, Qiang Hu, Hongxi Lu, Junxi Wang, Yi Luo, and Jinmin Li

Citation: Journal of Applied Physics 115, 123101 (2014); doi: 10.1063/1.4869336

View online: http://dx.doi.org/10.1063/1.4869336

View Table of Contents: http://scitation.aip.org/content/aip/journal/jap/115/12?ver=pdfcov

Published by the AIP Publishing

\section{Articles you may be interested in}

Phosphor-free nanopyramid white light-emitting diodes grown on $\left\{101^{-} 1\right\}$ planes using nanospherical-lens photolithography

Appl. Phys. Lett. 103, 241107 (2013); 10.1063/1.4840137

Selective area growth and characterization of InGaN nanocolumns for phosphor-free white light emission J. Appl. Phys. 113, 114306 (2013); 10.1063/1.4796100

Luminescent properties in the strain adjusted phosphor-free GaN based white light-emitting diode Appl. Phys. Lett. 93, 261117 (2008); 10.1063/1.3063044

Cool white III-nitride light emitting diodes based on phosphor-free indium-rich InGaN nanostructures Appl. Phys. Lett. 92, 261909 (2008); 10.1063/1.2952459

InGaN nano-ring structures for high-efficiency light emitting diodes

Appl. Phys. Lett. 86, 021101 (2005); 10.1063/1.1849439

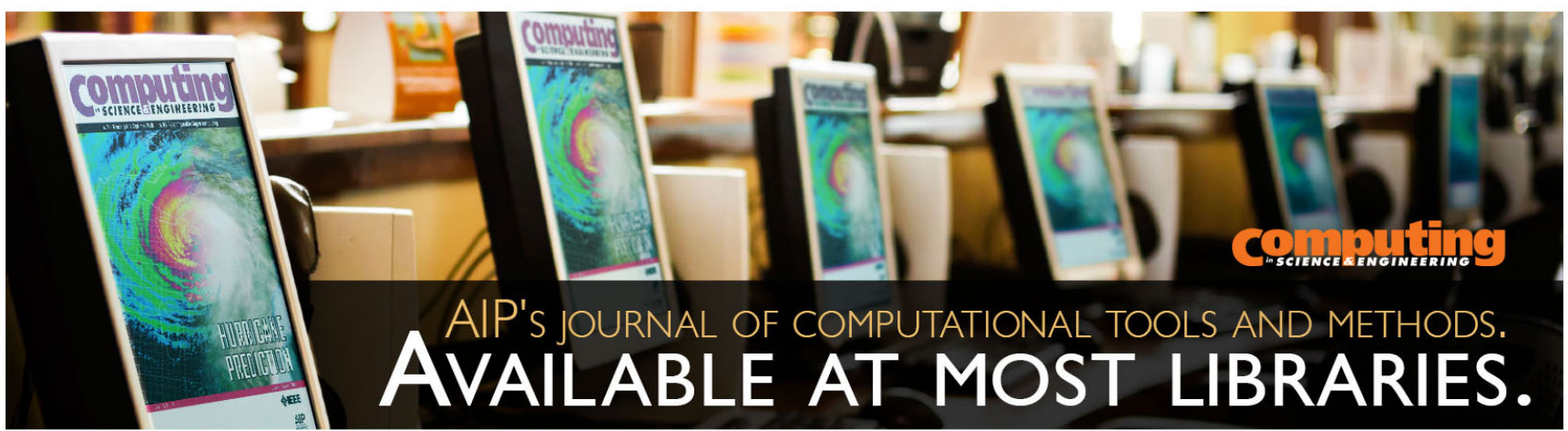




\title{
Fabrication and optical characteristics of phosphor-free InGaN nanopyramid white light emitting diodes by nanospherical-lens photolithography
}

\author{
Kui Wu, ${ }^{1,2}$ Tongbo Wei, ${ }^{1, a)}$ Haiyang Zheng, ${ }^{1}$ Ding Lan, ${ }^{3}$ Xuecheng Wei, ${ }^{1}$ Qiang Hu, ${ }^{1}$ \\ Hongxi Lu, ${ }^{1}$ Junxi Wang, ${ }^{1}$ Yi Luo, ${ }^{2}$ and Jinmin $\mathrm{Li}^{1}$ \\ ${ }^{1}$ State Key Laboratory of Solid-State Lighting, Institute of Semiconductors, Chinese Academy of Sciences, \\ Beijing 100083, China \\ ${ }^{2}$ Department of Electronic Engineering, Tsinghua National Laboratory for Information Science and \\ Technology/State Key Lab on Integrated Optoelectronics, Tsinghua University, Beijing 100084,China \\ ${ }^{3}$ National Microgravity Laboratory, Institute of Mechanics, Chinese Academy of Sciences, Beijing 100080, \\ China
}

(Received 22 November 2013; accepted 12 March 2014; published online 24 March 2014)

\begin{abstract}
A novel nanopattern technique of nanospherical-lens photolithography is introduced to fabricate the InGaN nanopyramid white (NPW) light-emitting diodes (LEDs) by selective area growth. Highly ordered NPW LED arrays are achieved after optimizing the growth conditions. It is found that the NPW LEDs vary from warm white light to cool with the increase in growth temperature. For the cool white NPW LEDs, the spectrum is similar to the conventional white LEDs obtained from the blue LEDs combined with yellow phosphors. The blue emission originates from the upper sidewalls of nanopyramids, and yellow light is mainly emitted from the lower ridges with respect to the base of nanopyramids. Furthermore, simulation shows that the light extraction efficiency of NPW LEDs is about 4 times higher compared with conventional ones, and the escape cone is as much as $85^{\circ}$ due to their three-dimensional nanopyramid structures. These observations suggest that the proposed phosphor-free NPW LEDs may have great potential for highly efficient white lighting. (C) 2014 AIP Publishing LLC. [http://dx.doi.org/10.1063/1.4869336]
\end{abstract}

\section{INTRODUCTION}

High-efficiency GaN-based white light-emitting diodes (LEDs) have already provided energy-saving and environmental benefits in a number of applications. ${ }^{1}$ To date, efficient InGaN LEDs are only available in the blue region. As a result, white LEDs are typically realized by the luminescence down-conversion techniques by using yellow phosphors, such as cerium-doped yttrium aluminum garnet. However, inherent wavelength-conversion loss (sometimes called quantum deficit or stokes shift) and degradation of phosphors, which would bring a relatively short life-time for LEDs, cannot be overcome by conventional $\lambda$-converter materials, because the wavelength-conversion loss is almost inevitable in nature. ${ }^{2}$ The current white LEDs are thereby limited by low efficiency, color rendering, and long-term reliability. ${ }^{3,4}$ Moreover, the efficiency of multiply quantum wells (MQWs) LEDs on $c$-plane suffers from strong polarization-related internal electric fields that limits radioactive recombination efficiency because of the low spatial overlapping of holes and electrons. ${ }^{5-7}$ Achieving phosphorfree monolithic white semipolar GaN LEDs is supposed to be of great interest as an ultimate way to meet the requirements of high efficiency white lighting applications. ${ }^{8,9}$ To date, approach to fabricate phosphor-free white LEDs by stacking blue and yellow emitting InGaN active layers on cpolar planes cannot be realized, because yellow-emitting LED is very inefficient especially for long wavelength

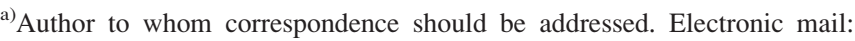
tbwei@semi.ac.cn. Tel.: +86-10-82305304. Fax: +86-10-82305245
}

LEDs. ${ }^{10-12}$ Another reason is that such a structure is lack of workable tunneling junctions. ${ }^{13}$ However, the epitaxial structure using selective area growth (SAG) technique is an effective way to achieve high-efficiency long wavelength emissions, ${ }^{5,14-18}$ and thus would be supposed to get monolithic phosphor-free white LEDs. Here, the QWs are grown on nonpolar/semipolar facets, where the facets can be tolerant for high Indium incorporation. Ascribed to strain relaxation at free surface of SAG, nonpolar/semipolar MQW LEDs possess low strain, ${ }^{19}$ nearly quasi-single crystal, $^{20}$ and reduced magnitude of piezoelectric field. ${ }^{21}$ Especially, the three-dimensional (3D) morphology offers them inherently large active areas and high lighting extraction efficiency. Using selective area growth method by metal organic chemical vapor deposition (MOCVD) on $n$-GaN templates grown on sapphire substrates, phosphor free white LEDs have been reported by utilizing hybrid structures of micro $\{0001\}$ planes and $\{11 \overline{2} 2\}$ nanopyramids, which are responsible for blue and yellow emissions, respectively. ${ }^{22}$ Nanocolumn, nanorod, and nanowire phosphor free white LEDs have also been demonstrated by high restrictive positional epitaxy growth of molecular beam epitaxy (MBE) on $\mathrm{Si}$ substrates. $^{23-26}$ However, there is still a long road to go before achieving effective and reliable phosphor-free white LEDs grown by SAG on self-assembled semipolar/nonpolar nanofacets. Highly ordered and homogeneous arrays with nanoscale semipolar/nonpolar facets, rather than the c-polar planes, are needed for the high-quality MQWs growth, leading to extremely large active areas and uniform electrical characteristics of large scale of individual nanopyramid LEDs. Commercial production also calls for mature 
technologies to achieve this nanopyramid LEDs including conventional MOCVD epitaxy, and well-established sapphire substrates.

In this paper, a novel nanopattern nanospherical-lens photolithography (NLP) technique, using self-assembled polystyrene nanospherical as convex lens ${ }^{27,28}$ for photolithography at conventional lithographic apparatus, is employed to fabricate nanoscale $\mathrm{SiO}_{2}$ masks on $n$-GaN templates as an alternative to expensive electron beam exposure technology. On these specialized templates, highly ordered nanopyramid LEDs with simple $\{10 \overline{1} 1\}$ facets are grown using MOCVD by SAG. Through optimizing the growth conditions, phosphor-free nanopyramid white (NPW) LEDs with simple $\{10 \overline{1} 1\}$ facets have been achieved, whose emissions are similar to blue LEDs combined with yellow phosphors. Subsequently, scanning electron microscopy (SEM) and transmission electron microscopy (TEM) measurements are performed to investigate the morphologies and structural properties of them. Cathodoluminescence (CL) and photoluminescence (PL) measurements have revealed the emission characteristics of NPW LEDs. Furthermore, finite-difference time-domain (FDTD) method is also used to simulate the lighting extraction efficiency of NPW LEDs. Potential high extraction efficiency is indicated by inspection of the 2D distribution of electrical field intensity and the time decay profile of the internal luminous energy.

\section{EXPERIMENTAL DETAILS}

Figure 1 showed the fabrication process of $\mathrm{SiO}_{2}$ masked $n$-GaN templates used for SAG. A $n$-GaN template consisted of $2 \mu \mathrm{m} n$-GaN and $2 \mu \mathrm{m} u$-GaN on sapphire substrate followed by a $400 \mathrm{~nm}$ thick $\mathrm{SiO}_{2}$ film. Then, $500 \mathrm{~nm}$ photosensitive resist (PR) AR-P 3120 was spin-coated on the top

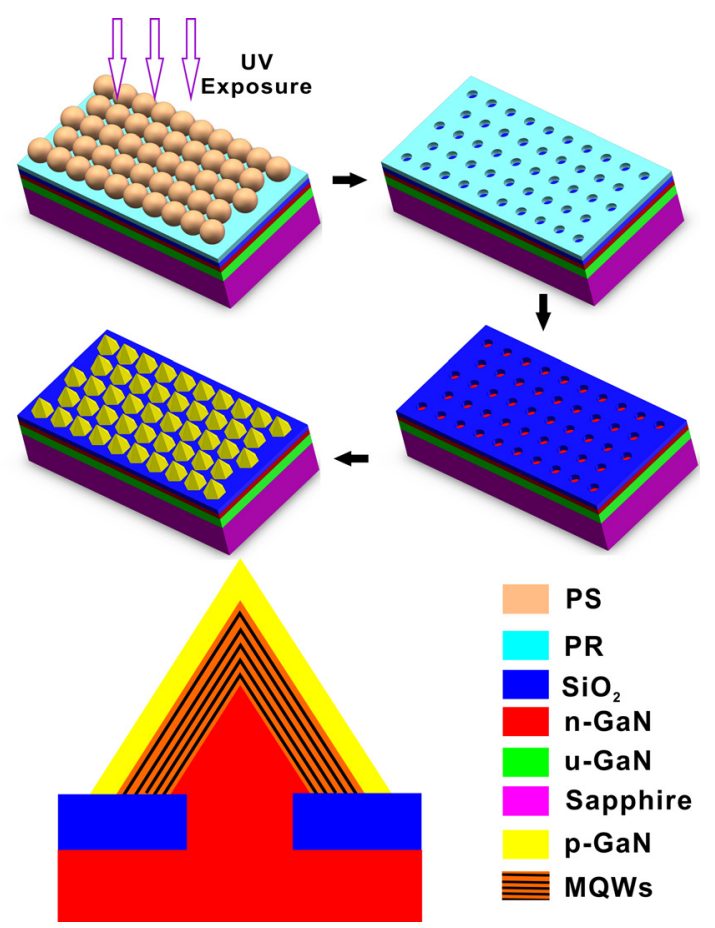

FIG. 1. Schematic illustrations of the fabrication process flow for phosphorfree nanopyramid LEDs by the NLP method. surface, before baking for $15 \mathrm{~min}$ at $95^{\circ} \mathrm{C}$ on hot plates. Subsequently, a close packed hexagonal polystyrene (PS) sphere monolayer layer was self-assembled on it by spin-coating. After UV exposure for $5 \mathrm{~s}$, developing about $5 \mathrm{~s}$, and ultrasonically cleaning off the PS nanospheres, a post-baking at about $110^{\circ} \mathrm{C}$ was used to improve the adhesion and the resistance of the resist structure which leaded to good etching stability. Circular openings with $400 \mathrm{~nm}$ diameter, and $900 \mathrm{~nm}$ period lattices were patterned and defined on $n$-GaN templates by inductively coupled plasma (ICP) etching, to expose the underlying $n-\mathrm{GaN}$ for SAG. In our study, the $n$-GaN templates were grown on $c$-plane sapphire substrates by MOCVD. Trimethylgallium, trimethylindium, trimethylaluminum, and ammonia $\left(\mathrm{NH}_{3}\right)$ were used as the source materials of $\mathrm{Ga}, \mathrm{In}, \mathrm{Al}$, and $\mathrm{N}$, respectively. The $n$-type and $p$-type doping sources are silane $\left(\mathrm{SiH}_{4}\right)$ and biscyclopentadienyl magnesium $\left(\mathrm{CP}_{2} \mathrm{Mg}\right)$. First, 4 min growth of $n$-GaN was selectively grown using $\mathrm{H}_{2}$ as carrier gas, with the growth temperature, pressure, and $\mathrm{V} / \mathrm{III}$ ratio to be $1050^{\circ} \mathrm{C}, 200$ millibars, and 12000 , respectively. On these growth conditions, the $n$-GaN on the nanoholes formed hexagonal nanopyramid with $\{10 \overline{1} 1\}$ facets. Five-period InGaN/GaN MQWs topped with $13 \mathrm{~min} p$-GaN cladding layers were also grown on these semipolar facets. For the $p$-GaN growth, the carrier gas was $\mathrm{H}_{2}$ and the growth temperature, pressure, and V/III ratio are $950{ }^{\circ} \mathrm{C}, 130$ millibars, and 6000 , respectively. As for the MQWs growth, the pressure of 150 millibars and V/III ratio of 3500 are defined, and the growth temperature was modified within the range of 720 to $780^{\circ} \mathrm{C}$, using $\mathrm{N}_{2}$ as carriers.

\section{RESULTS AND DISCUSSION}

PS nanospheres on the template topped with PR, as shown in Fig. 2(a), display the highly ordered arrays with hexagonal lattices. The right upper inset is the partial enlarged parts. PR and $\mathrm{SiO}_{2}$ nanoholes, after photolithography and ICP etching, are demonstrated in Figs. 2(b) and 2(c), with high magnification of cross section of PR pattern and $\mathrm{SiO}_{2}$ nanohole mask, respectively. The cross section of PR pattern, inset in Fig. 2(b), illuminates that the exposure and developing time is suitable for PR holes through. The evolution of $n$-GaN nanopyramid growth versus grown time is shown in Fig. 2(d). Here, the $n$-GaN nanopyramids were used for the subsequent MQWs growth. Figures $2(\mathrm{~d}-\mathrm{I})-2(\mathrm{~d}-\mathrm{V})$ correspond closely to the growth time of 1.5 , $2,3,4$, and $130 \mathrm{~min}$, respectively. Correspondingly, they are V-shaped, truncated, tip-planer, and well-shaped hexagonal pyramid. That is to say, 4 min growth is enough to make the tip-planer converge to zero, and have a perfect nanopyramid formed by $\{10 \overline{1} 1\}$ facets. The radius of the apex was determined to be less than $3 \mathrm{~nm}$, which comes to the limitation of SEM measurement. The perfect nanopyramids are beneficial to the MQWs to grow on the simple semipolar nanofacets. The mechanism of pyramid-forming results from that the $\mathrm{Ga}$ atoms of the lower part of a pyramid have longer migration length than the upper one. So, there are less Ga atoms arriving at the top of nanopyramids, resulting in low growth rate of the upper part. Surprisingly, neither the size nor the 

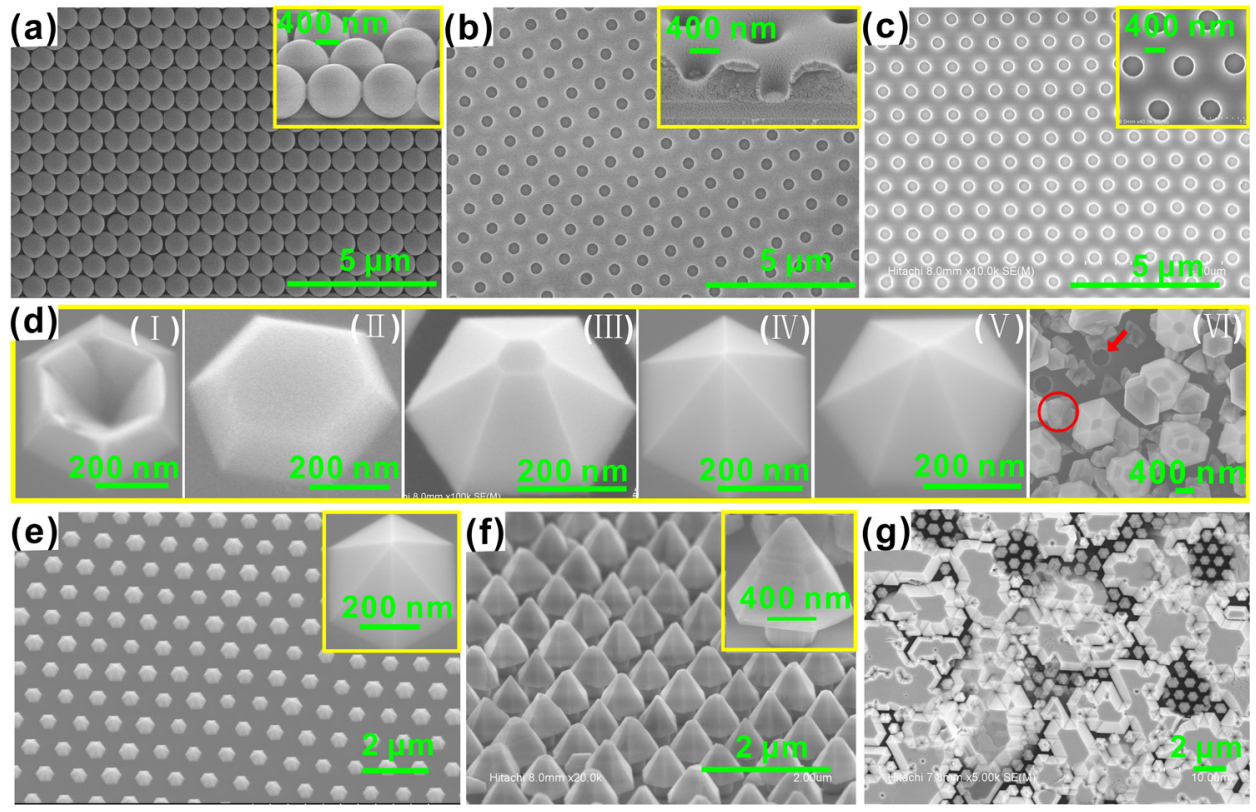

FIG. 2. Bird's-eyes view of SEM images of (a) highly ordered PS nanospheres on the $n$-GaN template topped with PR. The right upper inset is the partial enlarged image. Tilted SEM images of PR (b) and $\mathrm{SiO}_{2}$ (c) nanoholes, after NPW and ICP etching, respectively. High magnification of cross section of PR patterns and $\mathrm{SiO}_{2}$ nanohole masks are insetted, correspondingly. The growth evolution (d) of $n$-GaN nanopyramid, on which MQWs were grown, I-V corresponding to grown time of $1.5,2,3,4$, and 130 min. VI demonstrates $n$-GaN cannot be grown on such small $\mathrm{SiO}_{2}$ nanoholes (the red arrow), which is less than $200 \mathrm{~nm}$ in diameter, and deposits on $\mathrm{SiO}_{2}$ (red circle) being amorphous. (e) The $n$-GaN nanopyramids grown for 4 min, and the enlarged single one (upper right). (f) The nanopyramid LED arrays. The inset is a single nanopyramid LED after removing the $\mathrm{SiO}_{2}$ mask intentionally, for a good angle of view. The nanopyramid LEDs will collapse together (g), after long time of 30 min growth of $p$-GaN layer.

morphology of nanopyramids changes a lot, when the growth time was prolonged to $130 \mathrm{~min}$ in Fig. $2(\mathrm{~d}-\mathrm{V})$. That is because, at this particular growth condition of $n-\mathrm{GaN}$, the adsorption and decomposition rates come to a balance. However, $n$-GaN cannot nucleate in such small and deep $\mathrm{SiO}_{2}$ nanoholes (the red arrow in Fig. 2(d-VI) indicating), and being amorphous (the red circle indicating) on $\mathrm{SiO}_{2}$ masks. The highly ordered and homogeneous $n$-GaN nanopyramid arrays are shown in Fig. 2(e), and then the MQWs were grown on these exposed semipolar $\{10 \overline{1} 1\}$ nanofacets, followed by a $p$-GaN capping layer of 13 min growth. Then, the nanopyramid LEDs is completely fabricated. They are hexagonally packed arrays of nanopyramids with period lattice of $900 \mathrm{~nm}$, and the bottom of the pyramids is spaced about $210 \mathrm{~nm}$. All six facets are smoothed. Here, the $\mathrm{SiO}_{2}$ has been intentionally removed to show the mushroom shape of nanopyramid LEDs in Fig. 2(f). However, coalescence of the nanopyramid LEDs takes place as $p$-GaN growth time extends to $30 \mathrm{~min}$, which is maybe because $p$-GaN has relatively higher lateral growth rate than that of $n-\mathrm{GaN}$, in Fig. $2(\mathrm{~g})$. In our experiments, this coalescence is much avoided by optimizing the $p$-GaN growth procedure to keep the 3D morphology.

The optical characteristics of the nanopyramid LEDs were investigated by PL measurement. Figure 3(a) shows the room temperature PL spectra from the MQWs of nanopyramid LEDs. It is noticed that the MQWs, grown on planar and nanopyramid $n$-GaN templates at the same growth temperature of $720^{\circ} \mathrm{C}$, have a remarkable difference between their dominant peaks of the spectra. They are about $445 \mathrm{~nm}$ (blue regions) and $550 \mathrm{~nm}$ (yellow regions), which has depicted that the MQWs grown on semipolar nanofacets of $\{10 \overline{1} 1\}$ own much higher Indium content than that on c-plane templates. Moreover, the intensity of yellow regions (about $550 \mathrm{~nm}$ ) decreases evidently, while that of blue regions (about $438 \mathrm{~nm}$ ) increases, with increasing the growth temperature from 720 to $780{ }^{\circ} \mathrm{C}$. This change is likely because the QWs grown at high temperature leads to thermal degradation and thus more areas of semi-polar nanofacets attributing to short wavelength emissions. Interestingly, when grown at $780^{\circ} \mathrm{C}$, the nanopyramid LEDs exhibit the spectrum, which is similar to white LEDs from blue LEDs combined with yellow phosphors in Fig. 3(b). The PL spectrum from nanopyramid LEDs shows two emission peaks, which are at the blue and yellow regions. As shown in Fig. 3(c), EL measurements for NPW LEDs with a probe station also displays two emission peaks of blue regions (about $440 \mathrm{~nm}$ ) and yellow ones (about $550 \mathrm{~nm}$ ), which is consistent with the PL spectrum above. The inset in Fig. 3(c) shows the micro EL images taken from the nanopyramid LED at an injection current of $20 \mathrm{~mA}$.

In order to investigate the essential property of the NPW LEDs, cross-sectional transmission electronic microscopy (TEM) view of a single nanopyramid LED is also shown on the left upper part of Fig. 4(a). The nanopyramid LED has $600 \mathrm{~nm}$ height. The corresponding high-magnification scanning images demonstrate the clear alternately stacked bright and dark layers with respect to QWs and GaN quantum barriers (QBs), respectively. The left bottom part of Fig. 4(a) demonstrates that the facets form an angel of $62^{\circ}$ with respect to the c-plane, indicating the crystal planes to be $\{10 \overline{1} 1\}$. The arrow shows the c-axis $\langle 0001\rangle$ of the wurtzite structures. Due to the dislocation filtering effect of SAG, the nanopyramid LED is nearly perfect single-crystalline. The 

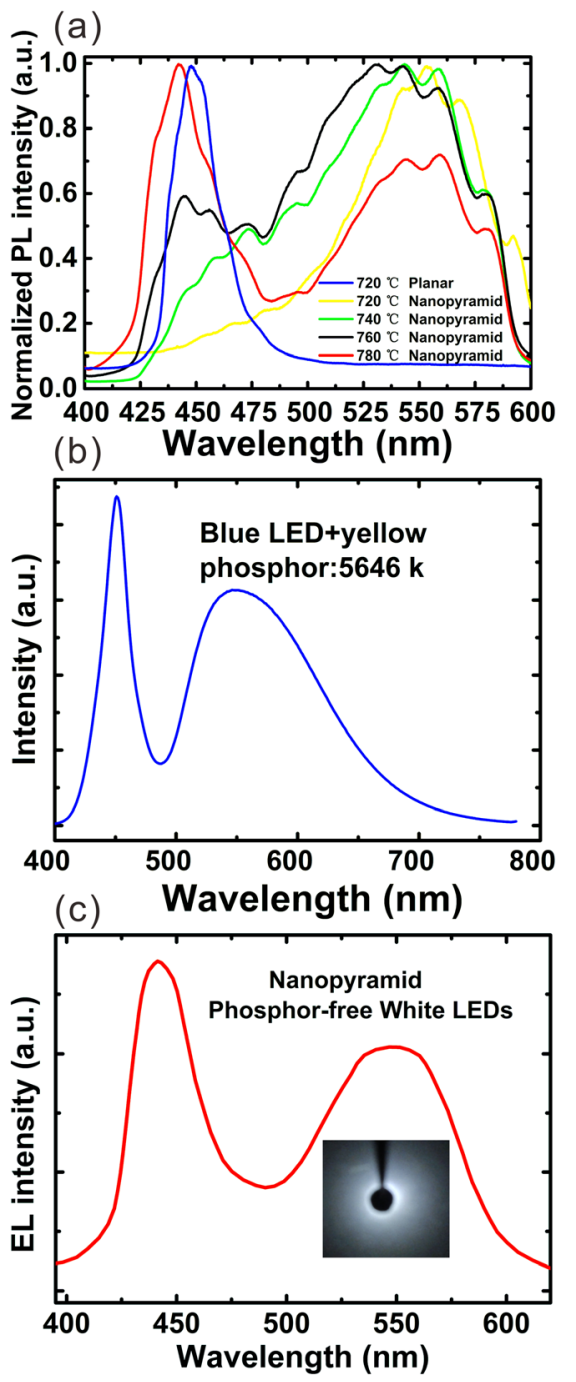

FIG. 3. (a) Room-temperature PL spectra of nanopyramid LEDs with MQWs grown on different temperatures, with reference of that grown on planar templates. (b) The spectrum of white LED from blue LED with yellow phosphors. (c) EL emission spectrum of NPW LEDs at a driving current of $20 \mathrm{~mA}$, the inset showing the corresponding optical microphotograph.

inset in Fig. 4(b) indicates the cross-section TEM image of a nanopyramid cut along the ridges. The QW width is observed with obvious width increment from the apex/base region. The normalized TEM-EDX (energy-dispersive Xray) line scan analysis was carried out along the given scan line of a (blue) and b (yellow), which were perpendicular to the QW and were located in the middle position of the upper part and the lower part of the nanopyramid, respectively, as shown in Fig. 4(b). It is interesting to see that the Indium composition for the scan line $\mathrm{b}$ is much higher than that of $\mathrm{a}$, as shown in the profile of Fig. 4(b). Figure 4(c) shows monochromatic CL images obtained at a wavelength of $440 \mathrm{~nm}$ and $550 \mathrm{~nm}$, at an accelerating voltage of $5 \mathrm{kV}$. It is noted that the MQWs on $\{10 \overline{1} 1\}$ nanofacets of nanopyramids emits blue lights from the upper of the sidewalls of nanopyramids, while the yellow emissions concentrate on the bottom ridges near the base of nanopyramids. The yellow emissions of the lower ridges may probably result from Indium atomic migration from the faces to the bottom ridges of nanopyramid LEDs. In addition, the wider QWs at the lower ridges
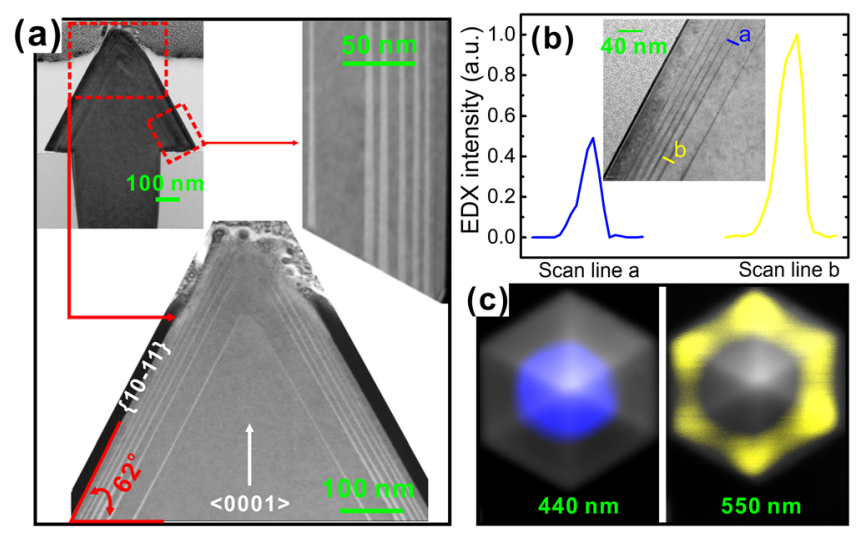

FIG. 4. (a) TEM cross-section of a single NPW LED and the corresponding of high magnification TEM images of the upper and the bottom parts. (b) For the cross-section cut along the ridges, the normalized TEM-EDX line scan analysis was carried out perpendicular to the QW, as indicated in the line a (blue) and b (yellow), representing the middle position of the upper part and the lower of the nanopyramid, respectively. (c) Monochromatic CL images at the given wavelength as on the images.

would bring long wavelength lighting. ${ }^{29}$ The short wavelength emissions of blue regions may be attributed to lattice pulling effects. ${ }^{30}$ When the $n$-GaN nanopyramids were growing, the strain generated and gathered in the apex areas. This effect leads to a lower Indium incorporation at the apex areas, yielding blue emissions. This spatial distribution of emissions is neither quite different from the MQWs on nanosized $\{11 \overline{2} 2\}$ facets, on which long wavelength emissions are from the upper faces of nanopyramids, and short ones are from lower parts, ${ }^{31}$ and nor different from the MQWs on $\{1 \overline{1} 00\}$ nanofacets, whose wavelength changes with the height. ${ }^{32}$ For microsize $\{10 \overline{1} 1\}$ pyramid facets, only one luminescence peak was displayed. ${ }^{6,33}$ The distinctive property is similar to Ref. 34, in which the nanowire was grown on c-plane along $\langle 10 \overline{1} 2\rangle$ direction, with $\{10 \overline{1} 1\}$ facets exposed outside. However, the brightness of the NPW LEDs is still weak, we believe that the brightness can be improved by optimizing the growth parameters, including the size, aspect ratio, and the structure of MQWs. ${ }^{35,36}$ Moreover, because the roughness surface impedes photoetching in pattern fabrication, the conventional device fabrication processes for planar thin-film LEDs needs improvement to be compatible with the NPW LEDs device. ${ }^{37,38}$ Our next work will focus on the device structures and chip processes to get detailed EL (electroluminescence) properties of those state-of-the-art NPW LEDs. Nevertheless, we can first use FDTD simulation to study the intrinsic nature of high light extraction efficiency of them.

To evaluate the effect of nano-pyramid structures on the light extraction of the NPW LEDs, three-dimensional (3D) FDTD simulations are carried out to numerically predict the light output power and far-field emission patterns. The NPW LED structures for FDTD calculations are simplified by reducing the thickness of the sapphire layer to $1 \mu \mathrm{m}$ and the lateral dimensions are limited to $11 \times 11 \mu \mathrm{m}^{2}$ to satisfy the memory limitation. Point sources are placed near the sample surface where the QWs are located. The emission wavelength of the source is set as dual wavelengths of $440 \mathrm{~nm}$ and $550 \mathrm{~nm}$ by referring to the EL measurements. Sufficient 
(a)
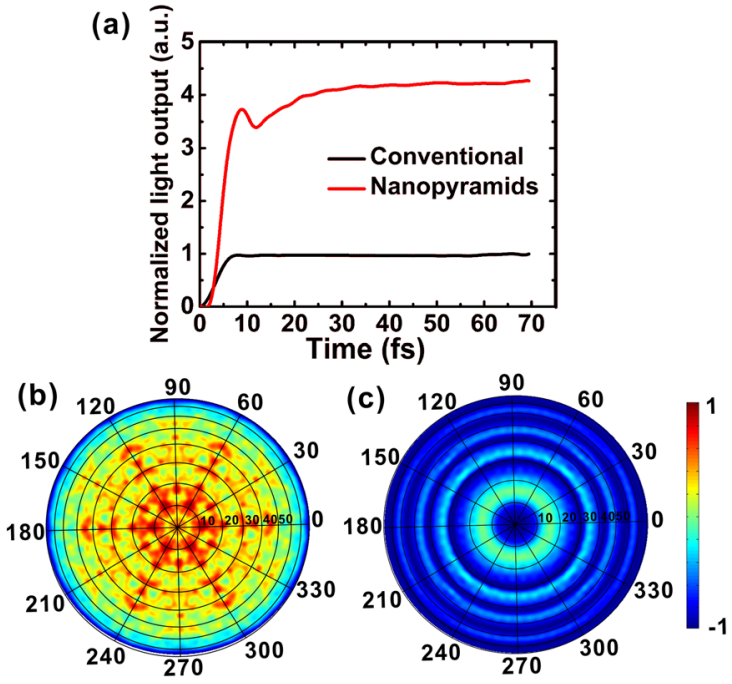

FIG. 5. (a) The light output power in NPW LEDs, with respect to the flattop one. Far-field distribution of light energy for the NPW LED (b) and the flat-top LED (c).

simulation time is required so that the light output signals obtain steady-state. The conventional LEDs with flat surface present a far-field pattern with perfect circular symmetry due to the fact that the total internal reflection occurs at the GaN/Air interface. Besides, as the light escapes from a very small angle (less than $40^{\circ}$ ), only a small part of the surface allows light to emit away. The inner and outer radiation ring caused by the Fabry-Perot effect is ascribed to the direct emission from the InGaN MQWs and the reflection from the bottom reflecting boundary, respectively. In contrast, when the nanopyramids structures are formed at the surface, a radiation pattern with hexagonal symmetry is accompanied by the light spreading over the entire surface, which indicates that the light escape cone is significantly broadened to over $85^{\circ}$, as shown in Figs. 5(a)-5(c). The simulated time-resolved normalized light output power in Fig. 5(a) shows about 4 times enhancement of light output power in NPW LEDs, when compared with the flat-top ones. However, in our simulation, it has 1.5 times improvement in the light extraction efficiency, for the LEDs with random surface roughening treatment to the LED top layers of Indium tin oxides (ITO) transparency conducting ones, compared to flat-top ones. This improvement of light output power in NPW LEDs can be explained by elimination of guided modes by incorporating the nano-pyramids, which have nanofacet 3D structures.

Figures 6(a) and 6(b) show the simulated emission pattern of the NPW and conventional LEDs, respectively. For the conventional LEDs, photons emitting outside the critical angle are seen to be totally reflected at the GaN/Air flat interface. Most of the light emitted from the active layer is trapped within the GaN layer. In contrast, the incorporation of periodic nanopyramid structures, onto the surfaces, is seen to suppress lateral guiding modes and redirect the trapped photons into radiated modes. Much of the light is directed outward, rather than undergoing multiple reflections within the GaN layer. Figure 6(c) shows a quantitative summary of the time decay of the normalized internal light energy of the NPW and conventional LEDs. Since higher internal light (a)

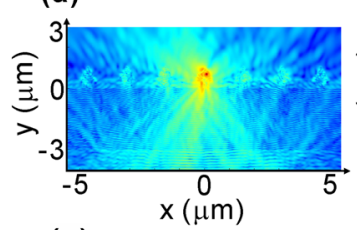

(b)

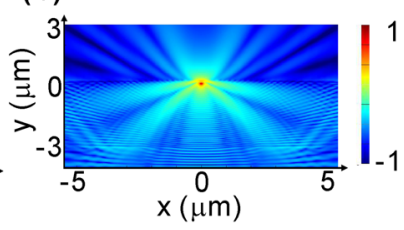

(c)

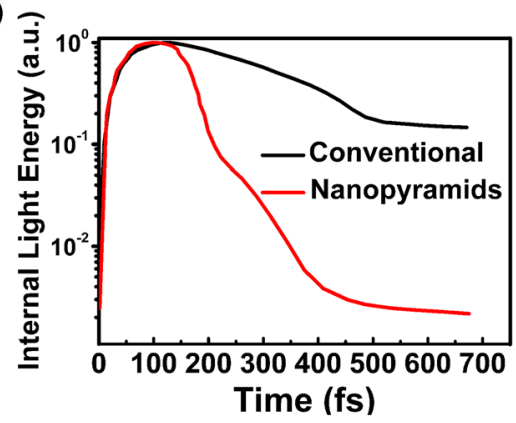

FIG. 6. The simulated emission patterns of (a) the NPW LED and (b) the conventional LED. (c) A quantitative summary of the time decay profile of the normalized internal light energy of NPW and conventional LEDs.

energy indicates the fact that more light remains trapped within the LED structures, the slowly decaying internal energy observed in the conventional LEDs indicates that the emitted light is accumulated within the structures due to the total internal reflections at the GaN/Air interfaces. In contrast, the internal energy in NPW LEDs rapidly decreases, which indicates much more light is extracted from the LED structures due to the reduced total reflections by incorporating the nanopyramids.

2D nanopyramids can help promote light extraction in LEDs via two possible mechanisms, which is similar to photonic crystal (PhC). If a photonic band gap (PBG) is opened up in the PhC, photons of guided modes are prohibited from lateral propagation in the band gap, only to radiate outward, and thereby light extraction efficiency is improved. ${ }^{39}$ However, such PhC does not possess a PBG along the plane since the lattice constant of the $\mathrm{PhC}$ is in the range far above the emission wavelength, ${ }^{40}$ but the lateral guiding mode can still effectively be suppressed by redirecting emission from guided modes into radiant ones ${ }^{41}$ over the range of the NPW LED operation frequencies. The periodic refractive index introduced by the $\mathrm{PhC}$ alters the propagation behavior of a photon, as described by the dispersion relation $\omega(\mathrm{k})$ with the light line $\omega=\mathrm{k}_{0} \mathrm{c}$ for free-space propagation. According to Bloch's theorem, the dispersion curves of Bloch modes are folded back at the Brillouin zone boundary. Therefore, the guided modes originally located below the light line are folded to be the diffracted modes located above the light line and thus can be extracted from the LED structures, in view of the lattice constant of the $\mathrm{PhC}$ is greater than the threshold value $\Lambda_{\text {cutoff }}$, given by $\Lambda_{\text {cutoff }}=\lambda /\left(\mathrm{n}_{\text {eff }}+1\right)$, where $n_{\text {eff }}$ is the effective index of the PhC layer. ${ }^{42}$ The lattice constants of the PhCs LEDs described in this study are in the range of $900 \mathrm{~nm}$ and thus satisfy this criterion.

\section{CONCLUSION}

In summary, a high-efficiency and low-cost nanopattern method of the NLP technique, was used to fabricate $\mathrm{SiO}_{2}$ 
opening masks on $n$-GaN templates. Through optimizing the conditions of SAG on the templates, a highly ordered hexagonal NPW LED arrays have been grown on $\{10 \overline{1} 1\}$ nanofacets. The spectrum are similar to white LEDs from blue LEDs with yellow phosphors, whose blue emissions are from the upper sidewalls of nanopyramids, and yellow lighting from the lower ridges with reference to the base of nanopyramids. By FDTD simulations, about 4 times enhancement of light extraction efficiency can be observed compared to conventional ones, and very high escape cone as much as $85^{\circ}$ can be predicted, attributed to the $3 \mathrm{D}$ nanopyramid structures which may have some meaningful suggestion of the later experiments. It's believed that these NPW LEDs will imply great potential to form the basis of a low-cost technique for future white lighting.

\section{ACKNOWLEDGMENTS}

This work was supported by the National Natural Sciences Foundation of China under Grant Nos. 61274040 and 51102226, the National Basic Research Program of China under Grant No. 2011CB301902, the National High Technology Program of China under Grant No. 2014AA032605 and Youth Innovation Promotion Association, Chinese Academy of Sciences.

${ }^{1}$ S. Pimputkar, J. S. Speck, S. P. DenBaars, and S. Nakamura, Nat. Photonics 3, 180 (2009).

${ }^{2}$ E. F. Schubert, T. Gessmann, and J. K. Kim, Light Emitting Diodes (Cambridge University Press, Cambridge, UK, 2006), p. 21.

${ }^{3}$ Y. Zhang, H. Zheng, E. Guo, Y. Cheng, J. Ma, L. Wang, Z. Liu, X. Yi, G. Wang, and J. Li, J. Appl. Phys. 113, 014502 (2013).

${ }^{4}$ C. C. Pan, T. Gilbert, N. Pfaff, S. Tanaka, Y. Zhao, D. Feezell, J. S. Speck, S. Nakamura, and S. P. DenBaars, Appl. Phys. Express 5, 102103 (2012).

${ }^{5}$ S. Srinivasan, M. Stevens, F. Ponce, H. Omiya, and T. Mukai, Appl. Phys. Lett. 89, 231908 (2006).

${ }^{6}$ M. Feneberg, F. Lipski, R. Sauer, K. Thonke, T. Wunderer, B. Neubert, P. Brückner, and F. Scholz, Appl. Phys. Lett. 89, 242112 (2006).

${ }^{7}$ C.-C. Pan, T. Gilbert, N. Pfaff, S. Tanaka, Y. Zhao, D. Feezell, J. S. Speck, S. Nakamura, and S. P. DenBaars, Appl. Phys. Express 5, 102103 (2012).

${ }^{8}$ T. Paskova, Phys. Status Solidi B 245, 1011 (2008).

${ }^{9}$ U. T. Schwarz and M. Kneissl, Phys. Status Solidi RRL 1, A44 (2007).

${ }^{10}$ T. Li, A. Fischer, Q. Wei, F. Ponce, T. Detchprohm, and C. Wetzel, Appl. Phys. Lett. 96, 031906 (2010).

${ }^{11}$ M. Ueda, K. Kojima, M. Funato, Y. Kawakami, Y. Narukawa, and T. Mukai, Appl. Phys. Lett. 89, 211907 (2006).

${ }^{12}$ S. Srinivasan, M. Stevens, F. Ponce, and T. Mukai, Appl. Phys. Lett. 87, 131911 (2005).

${ }^{13}$ M. L. Lee, Y. H. Yeh, S. J. Tu, P. Chen, M. J. Wu, W. C. Lai, and J. K. Sheu, Opt. Express 21, A864 (2013).

${ }^{14}$ Y. J. Hong, C. H. Lee, A. Yoon, M. Kim, H. K. Seong, H. J. Chung, C. Sone, Y. J. Park, and G. C. Yi, Adv. Mater. 23, 3284 (2011).
${ }^{15}$ S. P. Chang, Y. C. Chen, J. K. Huang, Y. J. Cheng, J. R. Chang, K. P. Sou, Y. T. Kang, H. C. Yang, T. C. Hsu, H. C. Kuo, and C. Y. Chang, Appl. Phys. Lett. 100, 061106 (2012).

${ }^{16}$ T. Kim, J. Kim, M. S. Yang, S. Lee, Y. Park, U. I. Chung, and Y. Cho, Appl. Phys. Lett. 97, 241111 (2010).

${ }^{17}$ C. C. Chen, C. H. Chiu, S. P. Chang, M. Shih, M. Y. Kuo, J. K. Huang, H. C. Kuo, S. P. Chen, L. L. Lee, and M. S. Jeng, Appl. Phys. Lett. 102, 011134 (2013).

${ }^{18}$ S. P. Chang, J. R. Chang, K. P. Sou, M. C. Liu, Y. J. Cheng, H. C. Kuo, and C. Y. Chang, Opt. Express 21, 23030 (2013).

${ }^{19}$ A. Romanov, T. Baker, S. Nakamura, and J. Speck, J. Appl. Phys. 100, 023522 (2006).

${ }^{20}$ R. Colby, Z. Liang, I. H. Wildeson, D. A. Ewoldt, T. D. Sands, R. E. García, and E. A. Stach, Nano Lett. 10, 1568 (2010).

${ }^{21}$ T. Wunderer, P. Brückner, J. Hertkorn, F. Scholz, G. J. Beirne, M. Jetter, P. Michler, M. Feneberg, and K. Thonke, Appl. Phys. Lett. 90, 171123 (2007).

${ }^{22}$ T. Kim, J. Kim, M. Yang, Y. Park, U.-I. Chung, Y. Ko, and Y. Cho, "Polychromatic white LED using GaN nano pyramid structure," Proc. SPIE 8641, 86410E (2013).

${ }^{23}$ M. Ghossoub, K. Valavala, M. Seong, B. Azeredo, K. Hsu, J. Sadhu, P. Singh, and S. Sinha, Nano Lett. 13, 1564 (2013).

${ }^{24}$ H. Sekiguchi, K. Kishino, and A. Kikuchi, Appl. Phys. Express 1, 124002 (2008).

${ }^{25}$ H. P. T. Nguyen, K. Cui, S. Zhang, M. Djavid, A. Korinek, G. A. Botton, and Z. Mi, Nano Lett. 12, 1317 (2012).

${ }^{26}$ W. Guo, A. Banerjee, P. Bhattacharya, and B. S. Ooi, Appl. Phys. Lett. 98, 193102 (2011).

${ }^{27}$ Y. C. Chang, S. M. Wang, H. C. Chung, C. B. Tseng, and S. H. Chang, ACS Nano 6, 3390 (2012).

${ }^{28}$ T. B. Wei, K. Wu, D. Lan, Q. F. Yan, Y. Chen, C. X. Du, J. X. Wang, Y. P. Zeng, and J. M. Li, Appl. Phys. Lett. 101, 211111 (2012).

${ }^{29}$ A. Kinoshita, H. Hirayama, M. Ainoya, Y. Aoyagi, and A. Hirata, Appl. Phys. Lett. 77, 175 (2000).

${ }^{30}$ G. Tourbot, C. Bougerol, A. Grenier, M. Den Hertog, D. Sam-Giao, D. Cooper, P. Gilet, B. Gayral, and B. Daudin, Nanotechnology 22, 075601 (2011).

${ }^{31}$ C. H. Liao, W. M. Chang, H. S. Chen, C. Y. Chen, Y. F. Yao, H. T. Chen, C. Y. Su, S. Y. Ting, Y. W. Kiang, and C. Yang, Opt. Express 20, 15859 (2012).

${ }^{32}$ J. R. Chang, S. P. Chang, Y. J. Li, Y. J. Cheng, K. P. Sou, J. K. Huang, H. C. Kuo, and C. Y. Chang, Appl. Phys. Lett. 100, 261103 (2012).

${ }^{33}$ H. Yu, L. Lee, T. Jung, and P. Ku, Appl. Phys. Lett. 90, 141906 (2007).

${ }^{34}$ S. K. Lim, M. Brewster, F. Qian, Y. Li, C. M. Lieber, and S. Gradecak, Nano Lett. 9, 3940 (2009).

${ }^{35}$ H. Sekiguchi, K. Kishino, and A. Kikuchi, Appl. Phys. Lett. 96, 231104 (2010).

${ }^{36}$ T. W. Yeh, Y. T. Lin, L. S. Stewart, P. D. Dapkus, R. Sarkissian, J. D. O'Brien, B. Ahn, and S. R. Nutt, Nano Lett. 12, 3257 (2012).

${ }^{37}$ M. Funato, T. Kondou, K. Hayashi, S. Nishiura, M. Ueda, Y. Kawakami, Y. Narukawa, and T. Mukai, Appl. Phys. Express 1, 011106 (2008).

${ }^{38}$ M. Funato, T. Kotani, T. Kondou, Y. Kawakami, Y. Narukawa, and T. Mukai, Appl. Phys. Lett. 88, 261920 (2006).

${ }^{39}$ N. Narendran, Y. Gu, J. Freyssinier-Nova, and Y. Zhu, Phys. Status Solidi A 202, R60 (2005)

${ }^{40}$ T. Oder, K. Kim, J. Lin, and H. Jiang, Appl. Phys. Lett. 84, 466 (2004).

${ }^{41}$ C. Wiesmann, K. Bergenek, N. Linder, and U. T. Schwarz, Laser Photonics Rev. 3, 262 (2009).

${ }^{42}$ Y. J. Lee, S. H. Kim, J. Huh, G. H. Kim, Y. H. Lee, S. H. Cho, Y. C. Kim, and Y. R. Do, Appl. Phys. Lett. 82, 3779 (2003). 